$32^{\text {nd }}$ European Modeling \& Simulation Symposium

$17^{\text {th }}$ International Multidisciplinary Modeling \& Simulation Multiconference

ISSN 2724-0029 ISBN 978-88-85741-44-7 @ 2020 The Authors.

DOI: $10.46354 /$ i3m.2020.emss.034

\title{
Selection of railway line segments that allow occupation by more trains based on simulation
}

\author{
Josef Bulíček ${ }^{1, *}$ and Michael Bažant ${ }^{2}$
}

${ }^{1}$ University of Pardubice, Faculty of Transport Engineering, Studentská 95, Pardubice, CZ-532 10, Czech Republic ${ }^{2}$ University of Pardubice, Faculty of Electrical Engineering and Informatics, Studentská 95, Pardubice, CZ-532 10, Czech Republic

*Corresponding author. Email address: josef.bulicek@upce.cz

\begin{abstract}
The paper is focused on research of railway infrastructure capacity assessment by computer simulation method. Specific goal of this paper is to discuss tools and possibilities for assessment, which segments of railway line can be divided into spatial segments for improving of capacity. This division can be done by using line blocks or more advanced systems, so called moving blocks, like ETCS L3 or interlocking and control systems based on on-line location of trains and controlling the gap between trains. The presumption is that equipment of a line segment between stations with such system costs considerable funds. Proposed method in this paper can help how to identify which segments have priority to be equipped or not. Simulation and transport technology points of view are combined in the paper.
\end{abstract}

Keywords: capacity; model; railway; simulation

\section{Introduction}

Basic condition of railway operation is that only one train can occupy one spatial segment of infrastructure (line) at a given time due to safety reasons. Initial division of a railway line is determined by the location of railway stations.

Line capacity can be limited due to this division. It is not an exception that line spatial segments between stations on regional lines are long about $10 \mathrm{~km}$. It means that one train can move on such part of railway line alone.

On the other hand, technical solution exists. Spatial segments can be divided on the line with blocks (block signals) that often work automatically (automatic block systems).

Current development is related to so called moving block - a system based on accurate localization of individual trains and on control of adequate distance between trains (moving in the same direction) at one track. ETCS L3 or some other interlocking and control system based on on-line satellite positioning of trains are currently in development.

Each blocking system dividing line segment into spatial segments costs considerable funds, so important decision is in which segment of line is such system effective from operational point of view. Possible low level of traffic can also be an important factor considered for decision about application because possible absence cannot be serious operational (capacity) limit.

Individual technical variants of line division to segments differ in volume of cost as well as in operational features. On the other hand, it has to be also reflected that some of technical variants are

(C) 2020 The Authors. This article is an open access article distributed under the terms and conditions of the Creative Commons Attribution (CC BY-NC-ND) license (https://creativecommons.org/licenses/by-nc-nd/4.0/). 
morally obsolete and the smart ones are still in development. Blocking system on the "middle level" will be considered in this paper - division of segment between 2 stations by one automatically working block (signals). This solution is common nowadays.

Core issue is to select where on a whole line (between which stations) it would be effective to equip the segment with blocking system. This will be noted as a "measure" in the rest of the paper.

No matter if the measure is planned for a long time or as a temporary measure (e.g. applied during maintenance works or until the moment when the line is completely upgraded). Operational features are similar. For that reason the topic is always actual.

Concept of operation is also a key aspect nowadays because quantitative features of capacity are often more important than qualitative ones. Sometimes it is not necessary to operate as many trains as possible but there is an effort to apply some advanced concepts in timetabling (e.g. using periodic intervals, supporting interchanges at different nodes etc.). Demand for trains is often lower than capacity, especially on regional lines.

The aim of the paper is to present first steps leading to a method for assessment - which line segments between stations are suitable to apply measure.

Simulation model created in the OpenTrack simulation software was applied for validation of theoretical presumptions for conducted research. Simulation is used in deterministic as well as stochastic conditions.

\section{State of the art}

Research focused on interlocking and controlling systems on railway is presented by (Quaglietta, 2013). There is an effort to minimize length of spatial segments as a tool for improving capacity. Similar solutions are often related to the need to improve capacity of dense-operated lines (including metro lines) with lack of free capacity.

Another similar topic is presented by (Vignali et al., 2020). It is focused on searching after optimized length of block sections (spatial segments) with application of ETCS L2 signalling system. The research is also based on simulation models in OpenTrack software.

Simulation techniques and aspects of simulation in the field of railway transport are often discussed topic as well. The paper (Rondón and Gomide, 2000) is an example. The paper is focused on dispatching of railway operation (in simulation model) with an effort to protect probability of occurrence of spatial conflict of trains (so called deadlock). These issues are not fully solved until now in the field of railway simulation.

Railway transport is also related to several optimizing tasks as well. Optimization of freight traffic in Iran is represented by (Yaghoni et al., 2015). Similar tasks are often complex and complicated with high computing demands. Fuzzy logic and genetic algorithm are applied in this case of Iran application (referred in mentioned paper).

Stability of railway operation is discussed by (Weiting et al., 2017). Operational risk index is the main result of that research. Influence of different operational disturbances is reflected.

Some topics are combining technical and operational points of view, for example (Arboleya et al., 2020). It is focused on modelling of electric-power supply systems on railway.

Demand after passenger transport is researched by (Kleprlík and Matuška, 2017).

Economical point of view on capacity can be represented by (Śiroký, 2017). It is focused on price for allocated infrastructure capacity as a tool for transport planning.

In general, application of simulation in the field of railway transport is common nowadays. Simulation is a powerful tool for this purpose because railway transport is complex and dynamic system with many relations and random elements.

\section{Materials and Methods}

This chapter is dedicated to proposal of new analytic indicators that have the ability to be applied within evaluation of effects of blocking systems. The first part is dedicated to definition of some basic concepts that are related to the issue. Next subchapter represents proposal of capacity indicators. The rest is dedicated to stochastic influences, including application of simulation.

\subsection{Background}

Application of blocking system that allows presence of more trains in the line segment is effective if there are more trains running in the same direction (see Figure 1 for illustration). Line segment is divided by block signal into 2 spatial segments. It is not possible to overtake or cross trains at the block signal.

Secondary, line block is effective when departure of the second train is located in defined time period of $T_{e}$ behind the first train (Figure 1). Dashed train (line) represents hypothetical situation without using a line block because the first train occupies whole line segment only for itself. Time $T_{e}$ expresses maximum time contribution (time saving) that can be reached by line block for this specific pair of trains. 


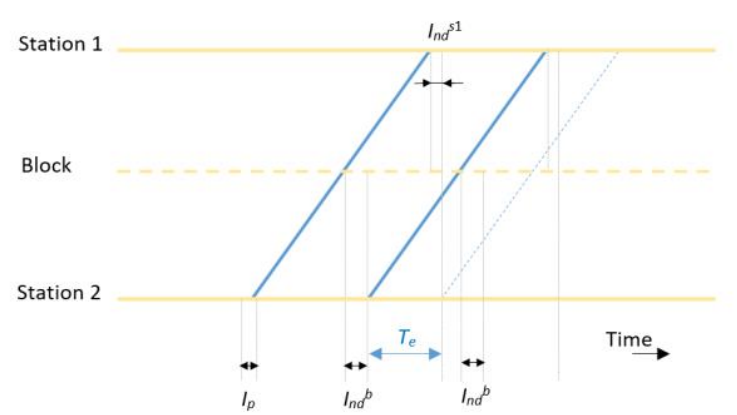

Figure 1. Time decomposition by using of line block. Source: Authors.

Period of $I_{p}$ corresponds to time needed for preparation for the next train. Periods of $I_{n d}$ represents needed time gap (interval) for preparation. The first one stands for the block $\left(I_{n d}{ }^{b}\right)$, the second one for the station $1\left(\operatorname{Ind}^{\text {s1 }}\right)$.

\subsection{Proposed capacity indicators}

Assessment of specific railway line starts with design of a timetable with applied measure (block) on the line.

The goal of this research is to propose some analytical capacity indicators that are able to validate discussed measures (division of line segments by blocking system).

Time expression of capacity is seen as perspective way for this assessment. Time of $T_{e}$ can be interpreted as a potential capacity increment as well.

The first proposed indicator (marked as CPS) is a count of planned situations that the second train departs in the period marked as $T_{e}$ (please see the Figure 1). The issue to be researched is that both trains can go later in reality so that this measure (e.g. block) can became ineffective when longer time interval between trains will occur. On the other hand, the measure can become effective for other couple of trains in stochastic conditions. The relation between CPS and stochastic reality (simulation) is assessed.

The second indicator (marked as TPS) is extended form of CPS focused on total planned time savings. The efficiency of measure can be different, because second train can be planned to different time positions (and not all are using $T_{e}$ in whole length). Principle of identification of time savings (marked as PS for one couple of trains) in Figure 2. TPS represents total expression for all trains (sum). In other words, PS corresponds to utilized part of possible capacity increase of $T_{e}$.

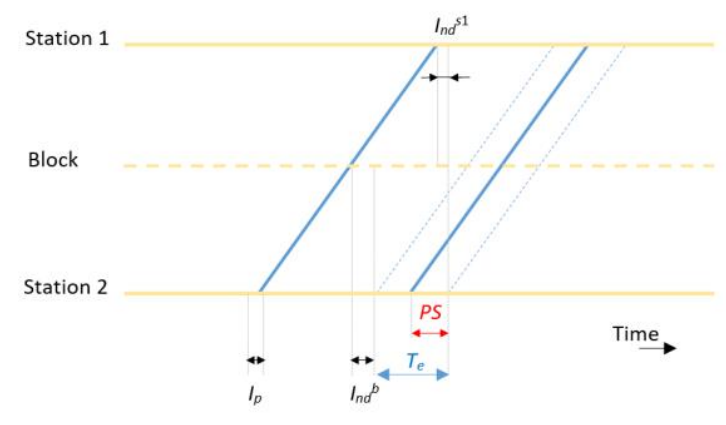

Figure 2. Utilized planned time saving Source: Authors.

There is one auxiliary indicator proposed as well. The indicator of $r$ is defined as ratio of planned time headway $(H)$ between 2 specific trains driving subsequently in the same direction to (minimal) headway allowed by interlocking using spatial segments divided with block $\left(H_{b}\right)$, see Formula (1).

$r=\frac{H}{H_{h}}$

Average value can be applied for evaluation of the line segment. For illustration of inputs to formula (1) see Figure 3.

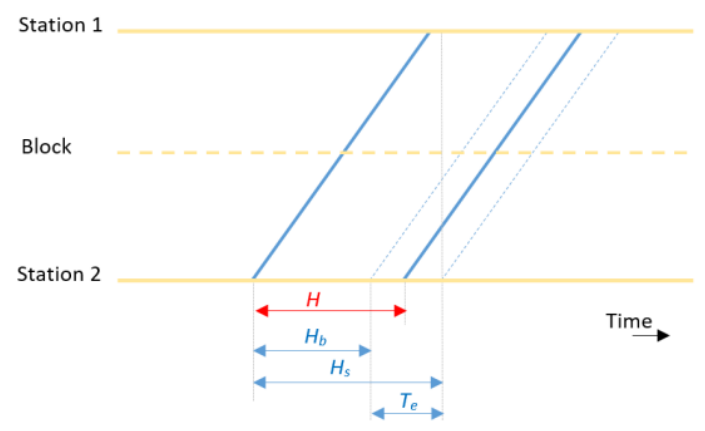

Figure 3. Headway between trains. Source: Authors.

The value of $H_{s}$ (in Figure 3 ) is for possible comparison with the state without blocking system. Specific values of all proposed indicators can be found in detailed analysis of timetable applied for solved line. This timetable has to reflect likely operation of a railway line with applied measure (using blocks or other systems dividing station segments into spatial segments).

\subsection{Stochastic influences}

Final effect of measure is related to stochastic influences in practice. Formula (1) is expression of probability $p_{b e}$ that the block is effective (applied) in the case that the first train is running according to timetable with no delay. This probability is based on the delay probability of the second train $f(t)$ as it is expressed in a Formula (2). In other words, it is a probability that the second train departs in period 
between moment of scheduled departure $T P_{s d}$ to the time moment of $\mathrm{TP}_{e}$ defined by the end of time period of $T_{e}$.

$P_{b e}=\int_{T P_{\text {sad }}}^{T P_{b}} f(t) d t=F\left(T P_{e}-T P_{s d}\right)$

\subsection{Simple simulation of blocking system}

Other point of view on efficiency of block can be illustrated within case study. Individual simulation of two trains in line segment equipped with block (signal) in the middle of segment. Time parameters are: $H_{s}=20 \mathrm{~min}, H_{b}=10 \mathrm{~min}, \mathrm{~T}_{e}=10 \mathrm{~min}$ (Figure 3). Both trains are considered in stochastic conditions. Probability of delay is set as $50 \%$. Specific values of delay (by delayed trains) are generated then by using exponential probability distribution with mean value of delay set on $5 \mathrm{~min}$.

Planned time headway of both trains is simulated in iterative way from $H=10 \mathrm{~min}$ to $H=40 \mathrm{~min}$ with step $\Delta h=1 \mathrm{~min}$ ( $\mathrm{x}$-axis in the Figure 4) and 10000 replications are computed for each iteration (variant of headway). Then there is computation how many times the block was effective (trains that were ready to depart in shorter time interval than $20 \mathrm{~min}$ ).

The results are in the Figure 4. The values of $H$ between 10 and 20 have $C P S=1$ (from 1 case) and TPS = $20-H$. When the value of $H$ is from 20 to 40 then CPS $=0$ and TPS $=$ omin.

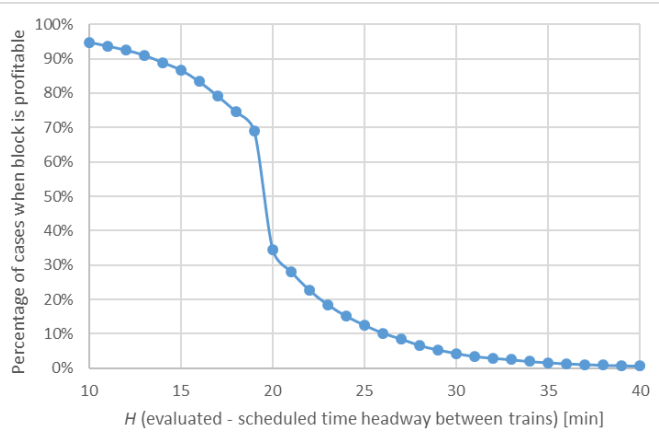

Figure 4. Percentage of cases when the block was effective (310,000 replications in total for 1 pair of trains). Source: Authors

Figure 4 as pilot simulation illustrates that the indicators of CPS and TPS are corresponding to the basic idea, but with some stochastic influences. In practical words, when the utilizing of block is presupposed by timetable (planned headway between 10 and $20 \mathrm{~min}$ ), there is also high rate of real cases of block utilization. It is between $70 \%$ and $95 \%$. In the rest of cases, practically occurred time intervals were longer, so that the whole line segment was clear by coming of $2^{\text {nd }}$ train (block ineffective). Relation between block efficiency and $T_{e}$ is visible as well (Figure $4, H$ from 10 to $20 \mathrm{~min}$ ).

\subsection{Complex simulation - scenarios}

The subchapters 3.3 and 3.4 were focused on some partial stochastic effects. Complex point of view can be found by using of model developed in the OpenTrack railway simulation software.

The research is conducted on the single-tracked railway line with the length of $50 \mathrm{~km}$ divided into 5 line segments. There were prepared 3 variants of infrastructure together with designed timetable. This creates 3 different simulation scenarios whereas modelled time period is 240 minutes in all 3 scenarios.

Basic timetable (also for scenario A) with any block contains 18 trains. Timetable consists of regional and long-distance passenger trains in both directions using whole the line. Some suburban trains are added to one of outer segments as well.

Scenario B has one block in the middle of the segment between $3^{\text {rd }}$ and $4^{\text {th }}$ railway station (i.e. in the middle of the whole railway line). The extent of operation is the same, but timetable is modified by planned using of block in the middle segment (CPS = $7 \mathrm{~min}, \mathrm{TPS}=24.5 \mathrm{~min}, r=1.0$ by all couples of trains following each other in the same direction).

There are 3 from 5 line segments equipped by block in the scenario C. Blocks are in the middle of segments and in both outer sections (before final stations). Extent of operation of both outer sections is different. One is used as suburban segment with heavy traffic with $C P S=8, T P S=28 \mathrm{~min}$, average $r_{\text {average }}=1.704$. The second is "occupied in standard way" by partial planned using of a block (CPS $=3$, TPS $=10.5, r=1.0$ by all cases). Middle segment (the third with block) represents other extreme situation - no regular using of block is planned (direction is switched by all couples of trains). Block is creating such kind of "reserve" in this case.

Stochastic mode of simulation was applied. There were realized 200 replications in all simulation scenarios. Other applied operation features (simulation parameters) are inspired by the reality of railway operation in the Czech Republic.

\section{Results and discussion}

The achieved results can be viewed from several angles. The following subchapters contain a discussion of the individual parts of the obtained results.

\subsection{Transport technology aspects}

Current effort is to create coordinated interchanges so that all trains (from all connected lines) arrive to the station just before defined time, mutual interchanges are possible and then the trains depart almost at the same order.

Traffic is organized for one end station in this way. Long-distance trains depart at X:03 and regional at 
$\mathrm{X}: 12$ without block (scenario A) due to the fact that the second train must wait until the first train comes to neighbour station. In the case of block in that outer segment (scenario 3) the second (more slowly) train is able to depart at X:08.5 (about 3.5min earlier). This can be considered as qualitative benefit for passengers.

Second important result is that both mentioned trains have the same time of arrival to the final station (on the opposite end of line) in both scenarios due to crossing on single-tracked line. This result is more or less subjective (depended on design of timetable), but it can be mentioned as an illustration that 3 blocks on the line may not ensure that the traffic become faster (fastening is in the first 2 segments before station of crossing).

Timetable in scenario B (block in the middle segment) is designed with an effort to make this block effective. It means that one train must follow the other in this segment). It causes impossibility to organize mentioned coordinated interchange at given station. Long-distance trains depart (arrive) at X:03 (X:01.5), regional at $\mathrm{X}: 43(\mathrm{X}: 41)$. This is not sufficient for changing passengers as well as for circulations of vehicles and train staff. Results are also subjective in specific case, but that this "risk" exists.

\subsection{Operational reliability in general}

Typical feature evaluated using simulation is reliability (or stability) of operation. This is expressed by delay and with increment of delay in simulated area (on the line). Overall results are in the Table 1.

Table 1. Results of stochastic simulation.

Source: Authors.

\begin{tabular}{lllll}
\hline \multicolumn{3}{c}{ Direction 1 } & \multicolumn{3}{c}{ Direction 2 } \\
\hline [s] & Delay & Increment & Delay & Increment \\
\hline Long-distance trains & & & \\
Scen. A & 183.6 & 28.2 & 147.3 & $-70,8$ \\
Scen. B & 278.8 & 126.2 & 61.2 & -156.9 \\
Scen. C & 179.4 & 26.6 & 94.0 & -94.1 \\
Regional trains & & & \\
Scen. A & 209.1 & 3.9 & 62.6 & -151.9 \\
Scen. B & 32.3 & -172.3 & 38.9 & -189.4 \\
Scen. C & 88.7 & -84.4 & 71.7 & -127.6 \\
Suburban trains & & & \\
Scen. A & 202.6 & -10.3 & & \\
Scen. B & 176.2 & -25.4 & & \\
Scen. C & 218.2 & 45.1 & & \\
\hline
\end{tabular}

Data for suburban trains in direction 2 are not missing, these trains are coupled with other trains in this segment.

The results can be discussed in following way. Installation of blocks can contribute to operation stability (many increment values are negative in scenarios B and C using block), but it is related also to timetable. For example, suburban trains have worse results in the scenario C. On the other hand, the extent of operation in suburban segment is heavy in this case.

\subsection{Waiting before line segments with block}

Second stochastic evaluation is focused on change of delay on the line segments in individual scenarios. The absolute values itself are not crucial, but it can be compared between the scenarios. Values are related to the subset of trains.

Table 2. Number of delay changes $>=30 \mathrm{~s}$ at the segment

Source: Authors.

\begin{tabular}{lllllll}
\hline \multicolumn{3}{c}{ Increments } & \multicolumn{4}{c}{ Reductions } \\
\hline Scen. & A & B & C & A & B & C \\
\hline AB & 146 & 154 & $\mathbf{1 0 0}$ & 553 & 199 & $\mathbf{5 0 8}$ \\
CD & 186 & $\mathbf{1 1 5}$ & $\mathbf{1 0 0}$ & 581 & $\mathbf{4 7 8}$ & $\mathbf{7 7 4}$ \\
EF & 179 & 124 & $\mathbf{1 6 1}$ & 263 & 434 & $\mathbf{1 7 8}$ \\
\hline
\end{tabular}

The result can be interpreted so that there are no serious relations between presence of block (bold values) and number of trains with important change of delay $(<=-30$ s or $>=30$ s).

\section{Conclusions}

The aim of the paper is fulfilled; different facts about relation between application of blocking systems into line segments and operation have been researched. On the other hand, it has not been confirmed that there are important relations between examined operational parameters and presence of block on line segment, but it can be seen that there are some relations between quality parameters and timetable itself. This will be a promising assumption that the relation can be found by further research conducted in more deep way. Application of simulation and OpenTrack software is adequate for research on similar topics.

\section{Funding}

The paper has been elaborated within the project "PosiTrans - Cooperation of the University of Pardubice will application sphere" granted by the European Union. Registration number: CZ.02.1.01/0.0/0.0/17_049/0008394. European Structural and Investment Funds: Operation programme Research, development and education. Authority: Ministry of Education, Youth and Sports of the Czech Republic.

\section{References}

Arboleya, P., Mayet, C., Mohamed, B., Aguado, J. A., and Torre, S. (2020). A review of railway feeding infrastructures: Mathematical models for planning and operation. eTransportation, 5 (2020): 100063

Kleprlík, J., and Matuška, J. (2017). The demand for public transport and modelling decision-making process of passengers. Transport Means 2017, 21 (2017): 197-20.

Quaglietta, E. (2013). A simulation-based approach for 
the optimal design of signalling block layout in railway networks. Simulation Modelling Practice and Theory, 46 (2014): 4-24

Rondón, M., and Gomide, F. (2000). Line block analysis in railway dispatch and simulation systems. IFAC Control in Transportation Systems, 2000: 389-393.

Široký, J. (2017). Price for the Allocation of Railway Infrastructure Capacity as a Tool for the Improvement of Train Transport Planning. MATEC Web of Conferences, 134 (2017): 00052

Yaghini, M., Momeni, M., Sarmadi, M., Seyedabadi, and Khosharftar, M. M. (2015). A fuzzy railroad blocking model with genetic algorithm solution approach for Iranian railways. Applied Mathematical Modelling, 39 (2015): 6114-6125

Vignali, V., Cuppi, F., Lantieri, C., Dimola, N., Galasso, T., and Rapagná, L. (2020). A methodology for the design of sections block length on ETCS L2 railway networks. Journal of Rail Transport Planning \& Management, 13 (2020): 100160

Weiting, Z., Ullrich, M., Yong, C., and Jiajian, L. (2017). Operational risk analysis of block sections in the railway network. Journal of Rail Transport Planning \& Management, 7 (2017): 245-262 